\title{
Le SIRH : Tableau de bord RH comme outil de pilotage et de prise de décisions
}

\author{
Chaali Kaoutar ${ }^{1}$, Bentahar Mohammed ${ }^{2}$ \\ ${ }^{1,2}$ FSJES-Tanger Université Abdelmalek Essaidi Maroc
}

\begin{abstract}
Résumé : Le système d'information des ressources humaines (SIRH) est devenu un outil incontournable des professionnels de la gestion des ressources humaines (GRH) en raison des volumes de traitement, de l'évolution permanente de la législation, des règles, et de la complexité des traitements. Il est la base pour l'élaboration d'un tableau de bord RH qui constitue pour les responsables RH et les décideurs un outil de pilotage et d'aide à la prise de décisions efficientes et efficaces basées sur des informations pertinentes.
\end{abstract}

Mots clés : le SIRH- Le tableau de bord RH- outil de pilotage - ressources humaines.

\begin{abstract}
The human resources represent for any company its main assets, therefore the human resources management is an index of the its performance. Considering that the functions of the human resources management are administrative processes of which, the quality can be analyzed, estimated and therefore improved. the use of data processing seems a compulsory and The creation of a dashboard of the human resources will allow the head manager, thanks to precise information of the company situation, to contribute more actively to the resolution of the major problems and to the permanent improvement of the company efficiency, thus to its economic success.
\end{abstract}

Keywords: the human resources information system- dashboard Human Resources- management tool- human Resources.

\section{INTRODUCTION}

Les seules armes concurrentielles dont disposent aujourd'hui les entreprises sont l'organisation et le capital humain. Dans un environnement de plus en plus compétitif où les évolutions technologiques et mondialisation guident les stratégies, la fonction du capital humain comme source de valeur ajoutée devient en effet primordiale. La meilleure illustration de ce fait réside dans la diffusion et le détail croissant des indicateurs RH. Administration du personnel, mesure de l'efficacité des processus RH, analyse des politiques RH... Les indicateurs RH, de plus en plus précis, se combinent pour offrir un matériau indispensable à la conception des stratégies de ressources humaines. Mobilité, rapidité des décisions, nouvelles législations, restructuration... Telles sont les contraintes complexes et plus nombreuses que doivent désormais intégrer les entreprises dans leur gestion quotidienne. Pour répondre à ces enjeux et assurer la réussite de leur organisation, l'utilisation d'outils adaptés, venant accompagner et supporter la mise en œuvre de nouvelles pratiques et de nouveaux processus de gestion des ressources humaines, apparaît comme la garantie d'un avantage compétitif réel. Le capital humain, véritable ressource vivante, impose aujourd'hui de passer d'une gestion du personnel classique à une gestion globale, dynamique et innovante pour mieux anticiper, choisir, accueillir, former, rémunérer, apprécier, communiquer, partager et décentraliser. La gestion de cette nouvelle dynamique est rendue possible par la mise en œuvre d'un système d'information RH qui produit la bonne information au bon moment et redistribue vers les différents services et niveaux hiérarchiques de l'entreprise. On le sait, aujourd'hui, communiquer est le principal intérêt retenu par la majorité des DRH pour mettre en place un SIRH. Communiquer, c'est d'échanger des indicateurs sociaux avec les acteurs des différents niveaux de l'entreprise. Que vous choisissez une solution informatique intégrée ou un progiciel de GRH plus classique, ce que vous recherchez surtout dans l'informatisation, c'est une application qui alimente les principaux processus RH et met à disposition l'information utile et indispensable à la bonne marche de la DRH au moment opportun.

Pour ce faire et pour faciliter la prise de décision, le gestionnaire a besoin d'information pertinente, tant financière qu'opérationnelle. Une visibilité accrue permet aux gestionnaires de mieux évaluer ce qui se passe et leur permet de réagir plus rapidement aux événements. Alors, ils ont besoin des instruments qui leur donnent des indications sur l'environnement et la performance de l'entreprise, et qui les aident à mettre le cap sur l'excellence. C'est le rôle des tableaux de bord. La construction d'un tableau de bord ressources humaines est une initiative qui permettra aux décideurs, grâce à des informations précises sur l'état de l'entreprise, de 
contribuer plus activement à la résolution des problèmes majeurs et à l'amélioration du rendement de l'entreprise et ainsi à son succès économique.

L'objectif de cet article est de répondre à la problématique suivante :

Dans le cadre d'une meilleure exploitation des ressources humaines d'une entreprise, Comment organiser et exploiter des informations concernant les RH d'une façon simple et claire afin d'avoir des indicateurs permettant de prendre des décisions efficientes et efficaces. La solution consiste à mettre en place des tableaux de bord RH, et ceci notamment à travers ce qu'on appelle un SIRH.

$\checkmark$ Donc Qu'est ce qu'un SIRH ?

$\checkmark$ Peut-on imaginer aujourd'hui gérer et développer les ressources humaines sans disposer d'un système d'information?

$\checkmark \quad$ A quoi sert un tableau de bord RH ?

Telles sont les principales questions auxquelles on va essayer de donner quelques éléments de réponses détaillés. Tout d'abord, nous expliquerons le concept de Système d'Information, cette notion étant à la base du terme de système d'information des ressources humaines. De plus, on ne peut pas comprendre la notion du SIRH sans avoir tout d'abord abordé l'histoire et l'évolution des ressources humaines. C'est pour cela que nous étudierons le passage de la fonction personnel à la fonction des ressources humaines.

\section{Le concept du SI :}

Le SI est une notion qui peut paraître abstraite, nous allons donc proposer une définition complète et claire. Pour cela, nous définirons séparément les deux termes qui composent le concept : « information » et « système ». Puis, nous analyserons son mode de fonctionnement afin de comprendre réellement son utilité.

\section{- L'information au cour de ce concept :}

Selon LAUDON K. et LAUDON L., « le terme information recouvre les données qui sont présentées sous une forme utile et utilisable par les personnes $»$.

Pour Jacques ARSAC ${ }^{2}$, « une information est une formule écrite susceptible d'apporter une connaissance. Elle est distincte de cette connaissance. ». Les informations nous permettent donc de compléter nos connaissances sur des événements, des personnes ou des objets. Elles peuvent exister sous plusieurs formes : écrites, picturales, orales ou sonores voire tactiles ou olfactives. En informatique, cette information est également appelée « donnée ». Elle va être conservée, traitée ou transmise à l'aide d'un support. Pour qu'une information soit fonctionnelle, il faut qu'elle soit utilisable et de qualité. Pour s'en assurer, elle doit remplir trois conditions ${ }^{3}$ : la dimension temporelle, le contenu et la forme.

- La dimension temporelle : l'information doit être régulièrement mise à jour et surtout être accessible et disponible à tout moment.

- Le contenu : l'information doit être fiable, exacte et précise. Elle doit provenir de sources sûres et vérifiées. Elle doit aussi être pertinente, adaptée et utile.

- La forme : L'information doit être claire. Son organisation doit permettre sa bonne compréhension. Cette condition a été facilitée grâce aux nouvelles technologies de l'information en permettant de les présenter sous des formes et supports variés. L'information au sein d'une entreprise possède de multiples sources. Elles peuvent être internes comme les documents comptables et financiers, les documents sociaux, les notes de services, les informations concernant les salariés ou bien externe comme les médias, les factures, la publicité, les lettres. L'information est donc considérée comme un outil de communication externe et interne, elle est très importante au sein des entreprises. L'information permet d'assurer la coordination des différents services qui composent l'entreprise. Elle contribue ainsi à la cohésion sociale. Les entreprises doivent donc mettre en place des moyens qui vont lui permettre une bonne gestion de la masse d'informations qui circule en son sein.

\section{- Qu'est ce qu'un système ?}

Nous venons de voir que les entreprises ont une multitude d'informations à gérer et qu'elles doivent mettre en place ce que l'on appelle un système d'information. Mais qu'est-ce qu'un système ? Pour Jean-Louis Le Moigne ${ }^{4}$ un système, c'est :

- Quelque chose : un objet réalisable appartenant au monde réel, et non à une idée.

- Dans quelque chose : Le système va interagir avec son environnement. On ne peut pas se concentrer sur une seule chose, il faut prendre en compte toutes les interactions.

\footnotetext{
${ }^{1}$ LAUDON, K., et LAUDON, J., Management des systèmes d'information, p. 14.

${ }^{2}$ ANGOT, H., Système d'information de l'entreprise.

${ }^{3}$ O'BRIEN, J., Les systèmes d'information de gestion.

${ }^{4}$ GILLET, M., GILLET, P., SIRH : Système d'information des ressources humaines.
} 
- Pour quelque chose : Il faut connaître la finalité poursuivie par l'organisation : recherche d'un profit ou un service à rendre.

- Fait quelque chose : L'activité de l'organisation va l'amener à sa finalité. Pour cela, il faut que l'activité crée de la valeur ajoutée. Le facteur de création de valeur est l'humain.

- Par quelque chose : Pour que l'homme soit performant, il faut structurer les actions des acteurs et faire circuler les flux nécessaires à ces actions.

- Qui se transforme dans le temps : L'action de création de valeur ajoutée évolue, notamment parce que c'est un processus de transformation de ressources.

Pour définir un système, on peut aussi s'appuyer sur la théorie générale de Ludwig

VON BERTALANFFY. Pour lui, ce sont quatre concepts qui définissent ce terme. 1

- L'interaction (ou l'interrelation) : Selon Edgar Morin, les interactions "sont des actions réciproques modifiant le comportement ou la nature des éléments, corps, objets, pour définir un système, on peut aussi s'appuyer sur la théorie générale de Ludwig

VON BERTALANFFY. Pour lui, ce sont quatre concepts qui définissent ce terme ${ }^{5}$

- L'interaction (ou l'interrelation) : Selon Edgar Morin, les interactions "sont des actions réciproques modifiant le comportement ou la nature des éléments, corps, objets, phénomènes en présence ou en influence."

- La totalité (ou la globalité) : Un système est un ensemble d'éléments, mais ce n'est pas que cela, les relations entre les données doivent aussi être prise en compte.

- L'organisation : L'organisation est le processus dans lequel les informations vont se regrouper, s'assembler et former une structure.

- La complexité : La complexité d'un système correspond à trois facteurs : le degré élevé d'organisation, l'incertitude de son environnement et la difficulté ou l'impossibilité d'identifier tous les éléments et de comprendre toutes les relations en jeu.

Qu'est ce qu'un système d'information?

Selon LAUDON K. et LAUDON L., un système d'information peut être de différents types ${ }^{6}$ :

- Manuel : c'est-à-dire qu'il repose sur l'utilisation du papier et du crayon,

- Parallèle : ceci représente le bouche à oreille,

- Informatisé : le SI repose sur des technologies informatiques, des logiciels et du matériel.

Lorsque nous parlerons de système d'information au cours de ce mémoire, nous ferons référence à ce dernier type de système : le système d'information informatisé. On pourrait donc penser qu'un système d'information représente un ensemble d'informations, mais c'est un peu plus compliqué puisque ce dernier est représenté comme la deuxième dorsale nerveuse après les hommes au sein d'une entreprise. Lorsque le cycle économique d'une entreprise est ralenti ou même arrêté, le gestionnaire de l'entreprise va chercher à trouver d'où viennent ces ralentissements (sous-payer ou sur payer les salariés, ne pas bien le gérer, le former ou bien le motiver, etc.),. Une fois les problèmes trouvés, il lui faut organiser des moyens pour être informé en temps réel de l'état d'avancement sur la résolution de ces derniers : c'est ce qui va constituer le système d'information. La définition de LAUDON K. et LAUDON L. du système d'information est la suivante : " un système d'information est un ensemble de composantes inter reliées qui recueillent de l'information, la traitent, la stockent et la diffusent afin d'aider à la prise de décision, à la coordination et au contrôle au sein de l'organisation $»^{7}$. Robert REIX ${ }^{8}$ s'est inspiré des théories de management pour donner sa propre définition de système d'information : " Ensemble organisé de ressources : matériel, logiciel, personnel, données, procédures... permettant d'acquérir, de traiter, de stocker des informations (sous forme de donnée, textes, images, sons, etc.) dans et entre des organisations ». Selon lui, le but d'un système d'information est d'«Apporter un soutien aux processus de travail dans l'organisation selon trois modalités principales : fournir de l'information, assister le travail humain et automatiser le travail ». Il ne faut pas penser qu'un système d'information ne soit qu'un système informatique, mais c'est tout système qui va permet de réaliser des processus de collecte, mais aussi de mémorisation et de traitement de l'information. Le SI est donc « un réseau complexe de relations structurées où interviennent des hommes, des machines et des procédures, qui a pour objet d'engendrer des flux ordonnés d'informations pertinentes, provenant de sources internes et externes à l'entreprise et destinées à servir de base aux décisions $\gg{ }^{9}$. Un SI se compose des ressources d'une organisation qui vont servir à récolter, structurer, saisir et stocker des données, pour, par la suite, transformer ces données en informations utilisables et les transmettre, les communiquer aux utilisateurs ou de les diffuser au sein de l'organisme sous une forme adaptée. Un système d'information ne doit pas être confondu avec un système

\footnotetext{
${ }^{5}$ VON BERTALANFFY, L., Théorie générale des systèmes.

6 LAUDON, K., et LAUDON, J., Management des systèmes d'information.

${ }^{7}$ LAUDON K. et LAUDON L. Management des systèmes d'information p.13.

${ }^{8}$ REIX, R., Systèmes d'information et management des organisations.

${ }^{9}$ ANGOT, H., Système d'information de l'entreprise.
}

DOI: $10.9790 / 0837-2202014559 \quad$ www.iosrjournals.org $\quad 47 \mid$ Page


informatique qui lui est composé d'ordinateurs, de programmes, de logiciels au sein d'une entreprise.La mission du système d'information est de gérer les informations opérationnelles et décisionnelles et donc de faciliter l'échange de ces informations entre les différents acteurs de l'entreprise. Il véhicule l'information au sein des organismes.

\section{- Le fonctionnement du SI:}

Le SI est en quelque sorte la mémoire de l'entreprise. Selon l'approche systémique ${ }^{10}$, l'entreprise peut se décomposer en trois sous-systèmes qui sont en perpétuelle interaction ${ }^{11}$ :

- le système d'opérations (modules opérationnels* MO): C'est là où s'effectuent les processus de production (actions pour la transformation de ressources en produits ou services).

- le système de décision ou de pilotage (modules pilotes* MP) : C'est ce système qui exerce un contrôle, une régulation, décision pour assurer la cohérence entre objectifs et les actions.

- le système d'information (SI) : C'est l'interface entre les modules pilotes et les modules opérationnels. Il enregistre, mémorise et traite les informations en provenance des MO, afin d'informer les MP. Ces derniers vont utiliser ces informations pour prendre des décisions d'action. Enfin, le SI renvoie ces décisions aux MO. Voir le schéma ci-dessous ${ }^{12}$.

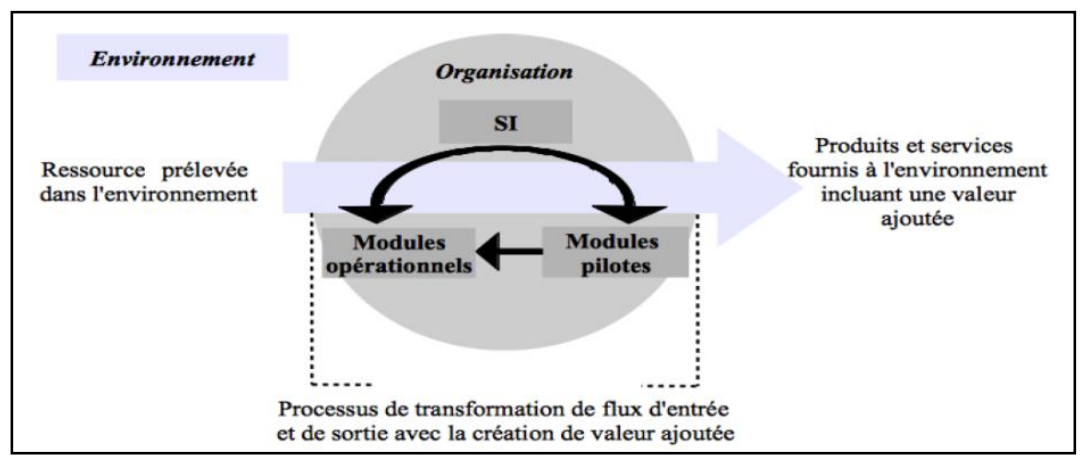

\section{Le concept de la fonction $\mathrm{RH}$ :}

On ne peut pas comprendre la notion de SIRH sans avoir auparavant définit le terme de ressources humaines. C'est pour cela, que nous allons effectuer un point sur les différentes mutations qu'a pu connaître le domaine des $\mathrm{RH}^{13}$. Ces différentes étapes de l'histoire vont nous aider à mieux comprendre pourquoi il y a eu une nécessité d'automatiser certaines tâches de RH, puisque selon D. Ulrich (2000), « Pour développer des activités à forte valeur ajoutée, les RH doivent nécessairement passer par une gestion efficiente, automatisée et par une instrumentalisation des processus ${ }^{14}$.Nous donnerons la définition ainsi que les missions de cette fonction. Puis, nous analyserons les facteurs principaux de cette évolution et donc du passage de la fonction du personnel à la fonction de Ressources Humaines.

\section{- Le terme de ressources humaines :}

La gestion des ressources humaines est une discipline assez récente. Elle est la dernière fonction née des grandes fonctions de l'entreprise après la production, la finance et le marketing.D'après Jean-Marc LE GALL, la DRH est une fonction de l'entreprise «qui vise à régir, à obtenir une adéquation efficace et maintenue dans le temps entre ses ressources (ses salariés) et ses emplois, en termes d'effectifs, de qualifications et de motivation. Elle a pour objet l'optimisation continue des compétences au service de la stratégie de l'entreprise, dans la définition de laquelle elle intervient $\gg{ }^{15}$. Le terme de gestion des ressources humaines englobe donc la gestion des relations individuelles de travail et des relations collectives de travail, et cela avec l'ensemble des salariés, des groupes de salariés et des organisations les représentants. Patrick ROUSSEL ${ }^{16}$ donne une autre définition

\footnotetext{
${ }^{10}$ GILLET, M., GILLET, P., SIRH : Système d'information des ressources humaines, p. 3-10 : Les fondements épistémologiques de la notion de systémique.

${ }^{11}$ Cours de Gestion des Processus et de la Qualité (GPQ).

${ }^{12}$ GILLET, M., GILLET, P., SIRH : Système d'information des ressources humaines, p.16 : Un système est composé de trois types d'éléments.

${ }_{13}^{13}$ JUST, B., Pas de DRH sans SIRH, p.17-29.

${ }^{14}$ Article de BAZIN, A ., « Nouvelles technologies et technologies mobiles : un levier de la performance organisationnelle et de développement du domaine $\mathrm{RH} / \mathrm{e}-\mathrm{RH}$ ? ».

${ }_{15} \mathrm{http} / / / \mathrm{www} . c e r c l e r h . c o m$.

${ }^{16}$ Patrice ROUSSEL est coordinateur de l'équipe de recherche au LIRHE, Laboratoire Interdisciplinaire de recherche sur les Ressources Humaines et l'Emploi, créé en 1995.
}

DOI: $10.9790 / 0837-2202014559 \quad$ www.iosrjournals.org $\quad 48 \mid$ Page


qui est la suivante : " La G.R.H. est l'ensemble des activités qui visent à développer l'efficacité collective des personnes qui travaillent pour l'entreprise. L'efficacité étant la mesure dans laquelle les objectifs sont atteints, la G.R.H. aura pour mission de conduire le développement des R.H. en vue de la réalisation des objectifs de l'entreprise. La G.R.H. définit les stratégies et les moyens en RH, les modes de fonctionnement organisationnel et la logistique de soutien afin de développer les compétences nécessaires pour atteindre les objectifs de l'entreprise. ». Pour lui, la GRH est donc une fonction, qui grâce à plusieurs actions et outils, va aider les employés à être plus efficaces dans le dessein d'atteindre, dans les meilleures conditions, les objectifs fixés par l'entreprise.

\section{- Évolution de la fonction : le passage de la fonction personnel à la fonction ressources humaines}

La fonction RH a connu beaucoup de mutations depuis son apparition. De plus, cette dernière n'a pas toujours été nommée ainsi. À ces débuts, on parlait plutôt d'une fonction du personnel et non de fonction RH. Pour comprendre ce passage, nous allons étudier les différentes étapes de cette évolution. On peut la scinder en sept grandes périodes ${ }^{17}$.

\section{a. La fonction du personnel:}

Durant la première période, c'est-à-dire de 1870 jusqu'au début XXème siècle, il n'y avait pas de fonction du personnel, du moins, elle n'était pas formalisée comme telle.En 1916, lorsque H. FAYOL énonce, ce qui est pour lui, les six grandes fonctions de l'entreprise, il ne mentionne pas la fonction personnelle ${ }^{18}$. Ceci notamment par le fait, qu'il existe peu d'entreprise dont la taille nécessiterait la mise en place de cette fonction. Dans les entreprises, le contremaître s'occupait lui-même de recruter les personnes et les formait de manière simple. La direction ou bien le patron dans les plus petites entreprises s'occupait de la gestion de la paie et de la comptabilité. Ce dernier pouvait être aidé par un comptable ou une secrétaire.C'est lors de la deuxième phase, durant la Première Guerre Mondiale, que le terme de fonction personnel va réellement apparaître. Les mauvaises attitudes des contremaitres et des responsables dans la gestion des hommes, le manque d'organisation, ainsi que les nombreux actes de violence sur les lieux de travail vont être à l'origine de multiples dysfonctionnements et d'une baisse de la productivité. À cela s'ajoutent des mouvements de réforme du courant de l'Organisation Scientifique du Travail et de l'école des relations humaines. Tout cela va avoir pour conséquences la modernisation de l'organisation du travail ainsi que la formalisation des méthodes de gestion du personnel. C'est à partir de là que la fonction personnel commence à naître. À la fin de la Première Guerre Mondiale, la réglementation sociale va évoluer avec la naissance de la négociation collective et de la création de l'assurance sociale. C'est aussi durant cette troisième période, que de nouveaux besoins dans le domaine de la gestion personnel vont se faire sentir notamment au niveau de la formation, de l'apprentissage et du recrutement.Lors de la quatrième phase située entre la fin de la Première Guerre Mondiale et le début de la Seconde, les entreprises sont confrontées à de nombreux problèmes sociaux et humains avec des vagues de grève. Au même moment, un renforcement syndical va s'effectuer avec la création des congés payés, de la semaine de 40 heures et de la mise en place de conventions collectives. Les premiers services du personnel commencent à se structurer, on assiste alors à l'émergence d'une fonction autonome.Les services du bien être se transforment en service du personnel avec les missions d'administrer les droits des salariés, de régler les problèmes de rémunération, de sélection, de formation, d'évaluation et de communication avec les syndicats. Cette fonction de personnel gère la main d'œuvre comme une variable d'adaptation dont le coût doit être minimum.On est encore très loin de la notion de ressources humaines. La dimension humaine s'efface devant l'objectif prioritaire que constitue l'optimisation de la production.Les 30 Glorieuses représentent la cinquième phase. Ces dernières vont être source de nouveautés aussi bien par de nouveaux textes de loi que par une nouvelle organisation du travail. Du côté de la réglementation, il y a la création du comité d'entreprise, la possibilité d'élire des délégués du personnel, la mise en place de la Sécurité Sociale ainsi que la création des ASSEDIC. Mais aussi des textes sur la liberté de négocier les salaires et le SMIG, le droit à une 3ème semaine de congés payés.Du côté travail, une certaine organisation va commencer à se mettre en place. On va essayer de simplifier certaines taches en appliquant de nouvelles méthodes. La fonction personnel va voir se professionnaliser la fonction de directeur du personnel.

C'est à partir de 1960, que la façon de penser va fortement évoluer et va entraîner l'apparition d'idées de réformes de l'entreprise. Cette évolution de la pensée a pour cause le meilleur niveau de qualification, mais aussi le fait que la sécurité de l'emploi ne suffit plus, il y a une volonté en matière de satisfaction du travail. Cette sixième période est marquée par les événements de mai 1968.La septième et dernière période connaît un ralentissement de la croissance mais aussi un fort développement de la concurrence internationale et des mutations technologiques. Durant cette période, la loi continue de s'enrichir (le bilan social, aménagement du temps de travail, loi Auroux) et va commencer à complexifier les différentes démarches que doivent suivre les

\footnotetext{
${ }^{17}$ JUST, B., Pas de DRH sans SIRH, page 17 à 21 : La GRH au fil du temps.

${ }^{18}$ Article de GUERIN, F., « La professionnalisation de la fonction RH ».

DOI: 10.9790/0837-2202014559 www.iosrjournals.org
49|Page
} 
directeurs du personnel. Les entreprises sont encouragées à avoir une productivité maximale ainsi qu'à la réduction de leurs coûts de production. Mais l'influence du modèle japonais se fait sentir notamment par le développement de la démarche participative, la décentralisation des décisions, l'autonomisation, la responsabilisation (culture d'entreprise, intéressement et participation) et les " 5 zéros » (défaut, délai, stock, panne, papier) où l'homme occupe un rôle central. L'homme devient alors un potentiel à mobiliser. C'est à ce moment que la fonction personnel laisse la place à la fonction ressources humaines et se décentralise auprès des acteurs de terrain, les managers. La fonction RH va alors voir le périmètre de ces actions s'élargir, elle va donc devoir être de plus en plus stratégique pour faire fasse à toutes ces évolutions.

\section{b. La fonction RH :}

Depuis l'émergence de la fonction des ressources humaines, cette dernière n'a cessé de s'affirmer comme une fonction «stratégique ». Ainsi BESSEYRE des HORTS (1987) dit que "la fonction [RH] elle-même devient majeure et acquiert le statut de grande fonction stratégique ». Pour PERETTI, « les DRH se retrouvent sur trois priorités : partage de la fonction ressources humaines, implication stratégique [...] et renforcement de son professionnalisme $»{ }^{19}$

Les actions d'investissement dans les hommes menées par les responsables RH doivent converger vers les objectifs de l'entreprise et de chacune des directions opérationnelles. Par conséquent, cette fonction est loin d'être réduite à un centre de coût. La fonction RH doit veiller à la bonne cohérence entre les objectifs RH et ceux de l'entreprise, elle doit définir des indicateurs et les suivre pour mettre en place, si besoin, des plans d'actions appropriés. Les objectifs des RH visent la stratégie globale de l'entreprise et les processus RH.

Selon Jean-Marie PERETTI, la fonction RH se trouve investie de quatre missions ${ }^{20}$ :

- Administrer efficacement : en optimisant ses propres processus, c'est-à-dire à diminuer les coûts de fonctionnement de sa fonction en mettant en place une organisation décentralisée de la fonction RH et une responsabilisation.

- Développer la motivation et l'implication des salariés : en assurant la présence et la gestion individualisée des compétences.

- Être un agent du changement : Il s'agit d'encourager les comportements nouveaux grâce à la formation et à certains programmes de mobilisation tels que de nouvelles modalités de travail ou la rémunération.

- Être un partenaire stratégique : c'est-à-dire être habile dans l'analyse et le traitement des données sociales, en disposant d'outils lui permettant d'apprécier l'impact humain. La stratégie pour Laurent BELANGER est : « un processus de formulation et de mise en ouvre des moyens appropriés en vue d'atteindre les objectifs d'une entreprise et de réaliser sa mission dans un environnement difficilement prévisible et concurrentiel ». ${ }^{21}$ Le DRH doit participer à la définition de la stratégie de son entreprise et doit veiller à la prise en compte en amont des conséquences RH des décisions prises.

Pour faire face à l'élargissement de son périmètre d'actions et aux évolutions rapides des lois, la fonction RH a un nouveau besoin important celui de la flexibilité.

C'est à ce moment que le SIRH va naître. Il va contribuer à l'optimisation de la fonction RH. Il va permettre de décentraliser certaines activités RH, et d'externaliser auprès de prestataires spécialisés les activités non stratégiques, permettant alors à la DRH de se recentrer sur les activités créatrices de valeurs ajoutées.

\section{Le SIRH :}

Il n'existe pas une seule et bonne définition du système d'information des ressources humaines, c'est pour cela que nous allons en étudier plusieurs pour réussir à formuler notre propre définition du SIRH.Tout d'abord, la définition de SIRH proposée par Bernard JUST ${ }^{22}$ qui est la suivante : «c'est un système informatique pouvant être constitué de différents logiciels hétéroclites communiquant entre eux par des interfaces techniques leur permettant d'échanger des données. Il est parfois constitué d'un unique système, on parle alors d'ERP (Entreprise Resource Planning) ou de PGI (Progiciel de Gestion Intégrée). Dans ce cas, les avantages sont multiples : il n'y a pas d'interface et les informations plus simples à restituer et à consolider.»Cette définition ressemble fortement à la définition donnée précédemment d'un système d'information. Pour introduire la notion de ressources humaines à cette définition, nous pouvons la compléter par la définition proposée par Bernard MERCK $^{23}$ : « un SIRH est un ensemble de logiciels plus ou moins interconnectés qui permettent d'assurer, de façon cohérente, différents actes administratifs et des opérations de gestion appliquées aux RH ». Cette définition introduit la notion de ressources humaines et montre qu'il y a un lien entre l'informatisation, c'est-à-

\footnotetext{
${ }^{19}$ Article de GUERIN, F., « La professionnalisation de la fonction RH ».

${ }^{20}$ PERETTI, J-M., Gestion des Ressources Humaines.

${ }^{21}$ BELANGER, L., PETIT, A., Gestion stratégique et opérationnelle des ressources humaines.

${ }^{22}$ JUST, B., Pas de DRH sans SIRH.

${ }^{23}$ MERCK, B., Equipes RH, acteur de la stratégie : L'e-RH : mode ou révolution?
}

DOI: 10.9790/0837-2202014559 $\quad$ www.iosrjournals.org $\quad 50 \mid$ Page


dire un ensemble de logiciels et les ressources humaines, et la gestion dédiée aux ressources humaines. Ce système en mettant en relation les actes administratifs et la gestion des RH se met au service du domaine des RH. La première définition introduit la notion de système, et la seconde la notion de ressources humaines, mais ces définitions n'évoquent pas réellement la notion d'information. La définition de Tanenbaum $(1990)^{24}$ introduit cette notion d'information et définit le SIRH comme " an HRIS as a system that will acquire, store, manipulate, analyze, retrieve, and distribute information about an organization's human resources » c'est-à-dire " un système permettant d'acquérir, de stocker, de manipuler, d'analyser, d'extraire et de distribuer des informations pertinentes au regard des ressources humaines d'une organisation ». Nous pouvons donc résumer la définition d'un système d'information des ressources humaines comme un ensemble de programmes ou d'applications informatiques permettant d'automatiser les différents processus qui composent la gestion des ressources humaines. Ce dernier couvre donc l'ensemble du domaine des RH. Le SIRH va avoir pour objectif d'aider le service des ressources humaines dans leur travail au quotidien. Notamment puisqu'il a permis d'automatiser des tâches administratives qui étaient répétitives c'est-à-dire la gestion de la paie, la gestion administrative, la gestion des temps, la formation, etc.

\section{- Structure d'un SIRH :}

Rappelons les deux remarques essentielles, qui sont à garder à l'esprit :

1) Le SIRH est une composante du SI de l'organisation. À ce titre, il est nécessaire de modéliser les points d'interaction entre le SIRH et les autres composants du SI.

\section{- Exemple :}

Dans une industrie, il y a une interaction forte entre le SIRH et la GPAO (gestion de production assistée par ordinateur). Les heures effectuées par le personnel ouvrier, les primes qui leur sont attribuées ou les absences, informations utilisées par la paie, émaneront de la GPAO.

2) Le SIRH est constitué d'une partie opérationnelle et d'une partie décisionnelle Comme c'est le cas pour la structure globale du SI de l'organisation, on rencontrera des aspects liés à la gestion de l'activité courante, par les modules opérationnels, et des aspects décisionnels, permettant aux modules pilotes d'étayer leurs prises de décisions.

-Les éléments composant la partie opérationnelle et la partie décisionnelle du SIRH sont représentés dans la figure ci-dessous.

-Cette figure présente les mêmes structures et principes dans le domaine RH que dans l'ensemble du SI.

Notamment, il existe :

- une interaction entre les composants opérationnels ou décisionnels (traits pointillés) ;

- une incidence des données opérationnelles sur la prise de décision, puis des décisions sur l'action opérationnelle (traits pleins);

- une alimentation du " reporting ", que constituent le bilan et l'audit social, par les autres composants, tant opérationnels que décisionnels (traits tirêts).

\footnotetext{
${ }^{24}$ Définition trouvée dans le mémoire suivant : Le Système d'Information des Ressources Humaines : un atout pour l'optimisation de la GRH au service de l'entreprise.

DOI: $10.9790 / 0837-2202014559 \quad$ www.iosrjournals.org $\quad 51 \mid$ Page
}




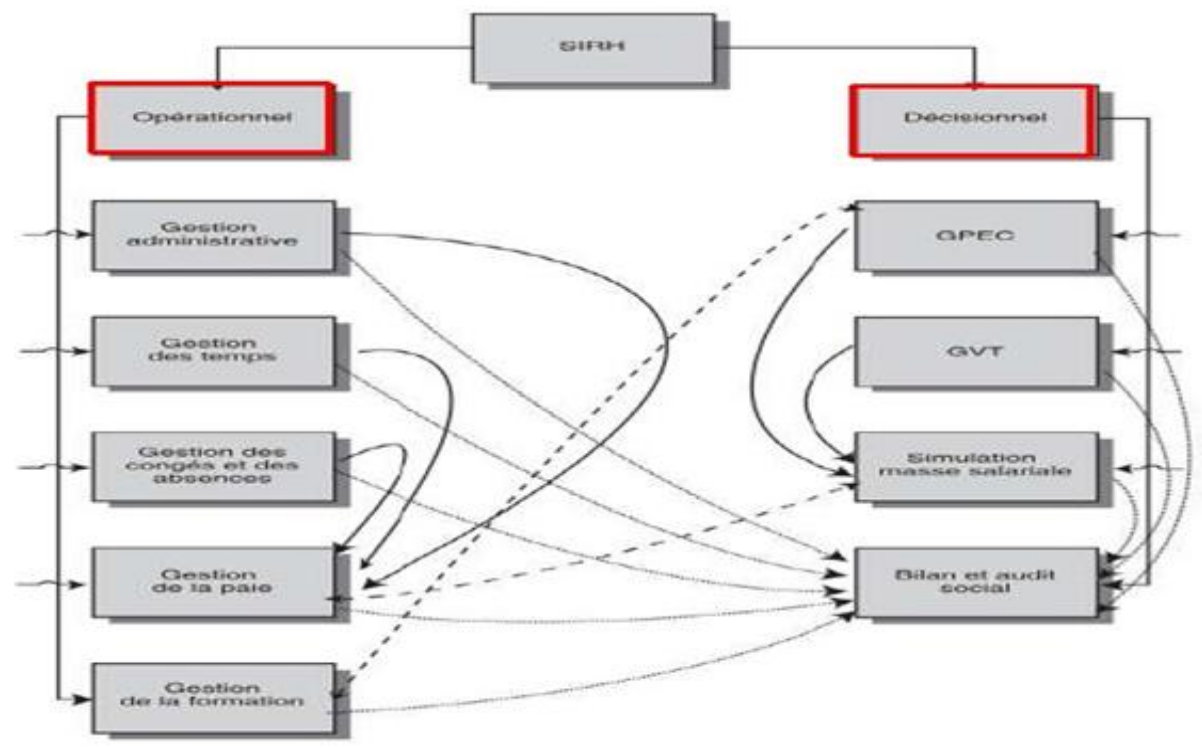

Source : SIRH, Michelle Gillet Patrick Gillet, édition DUNOD

\section{- Comment Choisir un SIRH ?}

Aujourd'hui, les entreprises qui se tournent vers les éditeurs de progiciels du marché, ont le choix entre plusieurs types de solutions.

\section{a. La gestion intégrée :}

La gestion intégrée vise à centraliser et coordonner les informations communes autour des ressources fonctionnelles. Les outils qui répondent à ce type de solution sont les Progiciels de Gestion Intégrés (PGI), aussi appelés ERP. Il s'agit de puissants logiciels destinés à gérer l'ensemble des processus opérationnels de l'entreprise, d'un domaine ou d'une fonction de l'entreprise. C'est une offre tout en un. Ce sont des outils complets, de plus en plus paramétrables, conviviaux et ergonomiques.

Certains ERP ne couvrent qu'un seul domaine, mais d'autres grands ERP peuvent couvrir plusieurs domaines. Les 2 buts principaux recherchés sont :

$\checkmark \quad$ Limiter les coûts de traitement en n'effectuant les mises à jour d'informations qu'une seule fois,

$\checkmark$ Coordonner les différents événements : par exemple, la vente d'un produit déclenche sa sortie de l'entrepôt, sa facturation

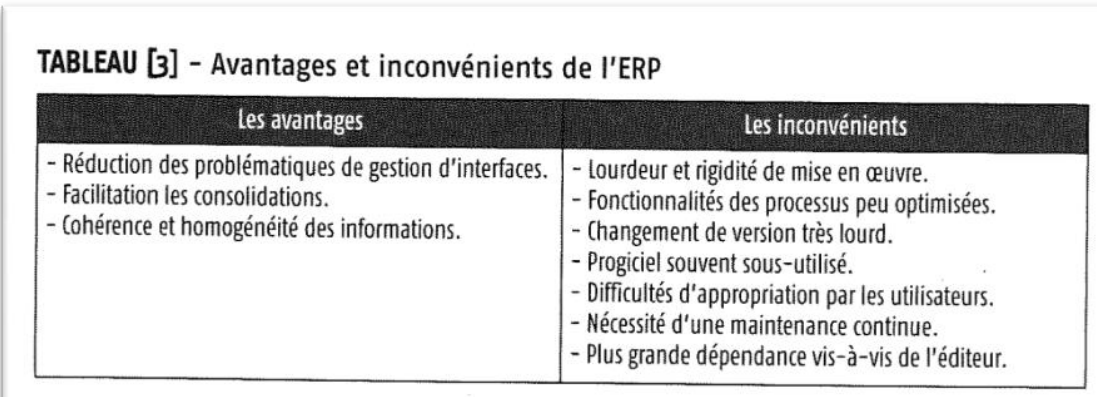

\section{b. La gestion par processus :}

La gestion par processus privilégie le but à atteindre. Il s'agit des solutions appelées Best of Breed.

C'est à partir du milieu des années 1990, que ces solutions de niche apparaissent. Elles apportent une réponse concrète, pratique et ponctuelle.

Lors de leur apparition, ces solutions ont eu du mal à s'imposer face aux ERP. Aujourd'hui, elles répondent à des besoins spécifiques des entreprises, et cohabitent donc fréquemment avec les ERP. 
TABLEAU [4] - Avantages et inconvénients duBest of Breed

\begin{tabular}{|l|l|}
\multicolumn{1}{|c|}{ Les avantages } & \multicolumn{1}{c|}{ Les inconvénients } \\
\hline - Alignement du SIRH sur la stratégie de la DRH. & - Gestion de plusieurs interfaces \\
- Plus de flexibilité. & - Pas de communication entre les différents outils. \\
- Réduction des développements spécifiques. & - consolidations possibles mais à développer. \\
- Rapidité de mise en œuvre. & - Multiplication des licences. \\
- Accroissement des niveaux de service. & \\
- Réduction des coûts. & \\
\hline
\end{tabular}

\section{c. L'informatique distribuée :}

L'informatique distribuée permet à une application de découvrir les applications qui l'entourent et qui sont autant de services autonomes auxquels elle peut s'adresser.

Il existe plusieurs types de solutions :

\section{$\checkmark$ L'ASP : Application Service Provider :}

En français les FAH, pour Fournisseurs d'Applications Hébergées, sont des entreprises qui fournissent des logiciels ou des services informatiques à des clients au travers de réseaux (internet en général). Il s'agit de l'externalisation de l'hébergement d'une application ou d'un service en ligne.

L'application logicielle est installée sur le système informatique du fournisseur et est accessible par l'utilisateur à travers un navigateur Internet ou grâce à un logiciel client délivré par le fournisseur.

\section{$\checkmark \underline{\text { Le SaaS : «Software as a Service» }}$}

Aussi appelé AH en français, pour Application Hébergée. Il s'agit d'une technologie consistant à fournir des services ou des logiciels informatiques par le biais du Web et non plus dans le cadre d'une application bureau ou client serveur.

Ce concept date du début des années 2000, et prend la suite de l'ASP. Le SaaS est un modèle économique qui consiste à commercialiser un logiciel non pas sous la forme d'un produit (en licence définitive), que le client installerait en interne sur ses serveurs, mais en tant qu'application accessible à distance comme un service, par le biais d'Internet et du Web. Dans ces applications de type « clients légers », les clients ne payent pas pour posséder le logiciel, mais pour l'utiliser.

Alors que l'ASP traditionnel se limite à la fourniture d'applications en mode hébergé, le SaaS se propose de donner accès à une plate-forme applicative modulaire, permettant d'y inclure également des développements spécifiques.

\section{$\checkmark$ Le SOA : Services Oriented Architecture}

Une architecture orientée services est une architecture logicielle s'appuyant sur un ensemble de services simples. L'objectif d'une architecture orientée services est donc de décomposer une fonctionnalité en un ensemble de fonctions basiques, appelées services, et de décrire finement le schéma d'interaction entre ces services. L'idée sous-jacente est de cesser de construire la vie de l'entreprise autour d'applications, pour faire en sorte de construire une architecture logicielle globale décomposée en services correspondants aux processus métiers de l'entreprise. C'est une évolution de l'informatique facilitant la communication de logiciel entre eux.

Cette architecture s'appuie sur les technologies Internet. Elle permet d'agréger et de croiser des informations provenant de différentes applications, et ainsi d'enrichir l'information.

\section{- Les fonctionnalités d'un SIRH}

Il existe une diversité des représentations d'un système d'information ressources humaines selon les auteurs RH, les consultants, les éditeurs RH. Nous retenons donc dans l'analyse de ces fonctionnalités du SIRH le découpage en 8 processus à savoir :

\section{La paie :}

La paie est un « ensemble de processus, de pratiques et d'applications mis en ouvre pour calculer les éléments de paie, effectuer les paiements et s'assurer de la conformité réglementaire de l'activité. Cela couvre toutes les opérations entrant dans la production de la paie : calcul des salaires, des rémunérations, des avantages et des déductions, mais aussi l'édition et la distribution des bulletins, les déclarations aux organismes tiers ainsi que la maintenance légale ». La gestion de la paie va regrouper toutes les informations traitées précédemment. 
Chaque information a des répercutions différentes sur la paie du salarié.Selon Bernard JUST, la paie est « le processus le plus abouti dans l'entreprise, pour_la simple raison qu'elle est vitale à la stabilité de la "paix sociale » devenant ainsi essentiellement indispensable ». Au début, la paie été au cœur du SIRH, mais maintenant c'est le dossier du salarié, comme le montre Bernard JUST : « le dossier individuel est le socle de tous les processus SIRH ».

Face à la complexification de la paie notamment dû aux évolutions légales et réglementaires, les éditeurs de solutions SIRH doivent sans cesse adapter leur logiciel aux nouveaux besoins.

\section{La gestion administrative du personnel :}

Cette gestion a pour objectif de traiter, de manière qualitative, les informations concernant le salarié et qui sont conservées dans le dossier de celui-ci. Ces informations sont multiples et variées comme le montre la liste suivante, qui n'est pas exhaustive, elle évolue en fonction des règlements et du contexte de l'entreprise et de son environnement.

- Gérer les congés,

- Gérer les absences ainsi que leurs incidences diverses,

- Gérer les visites médicales,

- Gérer les titres de séjour pour les étrangers,

- Contrôler le droit à exercer certaines tâches,

- Gérer les obligations par rapport aux travailleurs handicapés,

- Gérer les tickets restaurant, chèques vacances ou titres de transport.

La mise en place du SIRH va permettre d'améliorer la qualité du traitement des données administratives, notamment par la diminution des délais de transmission des informations, des erreurs de recopie et des omissions dans le traitement des données, grâce aux alertes de renouvellement.

3. La gestion des temps et des activités :

La Gestion des temps et des activités constitue l'enregistrement quotidien de l'exécution par le salarié de sa part de la transaction salariale, autrement formulé l'enregistrement de son temps de travail effectif. La GTA est soumise à la réglementation du temps de travail pour affecter dans différents compteurs les données de présence et d'absence qu'elle enregistre, et les convertir en droits : congés (minimum cinq semaines par an, plus journées obtenues suite aux accords d'aménagement et de réduction du temps de travail), temps de travail légal (plafonds journalier, hebdomadaire, mensuel et annuel)...

Elle propose aussi la planification du travail dans le temps (horaires de référence, exceptions, planification des rotations d'équipes : par exemple en $3 \times 8$, et aussi congés, compte épargne temps, etc...) mais également dans l'espace, quand un salarié exécute son contrat sur plusieurs lieux de travail possibles.

Ces enregistrements permettent la mesure du temps de travail effectué et donc de suivre les indicateurs : absentéisme, heures supplémentaires, astreintes...

4. La Gestion prévisionnelle des emplois et des compétences :

Le SIRH facilite la gestion prévisionnelle des emplois et des compétences puisqu'il va permettre de :

- Faciliter le travail de recherche d'une personne, qui appartient à l'entreprise ou qui figure sur les candidatures et qui possèderait le profil recherché.

- Définir les caractéristiques d'une offre,

- Mesurer les écarts entre les compétences d'une personne et les exigences du poste qu'elle occupe, afin de ne pas avoir de personne sur ou sous profilé par rapport au poste occupé,

- Préparer les différents entretiens et le plan de formation par rapport aux écarts.

- Donner un accès au salarié pour qu'il mette à jour les données le concernant (CV, demande de formation, etc.).

\section{Le recrutement:}

Les outils proposés par les éditeurs sont généralement peu interfacés avec le SIRH principal. Ce sont principalement des outils de niche qui assurent la gestion du recrutement en ligne.

Le processus présente une succession d'étapes :

- Les recruteurs déposent leurs offres sur l'espace emploi prévu dans un espace dédié d'un logiciel $100 \%$ web, disponible $7 \mathrm{j} / 7,24 \mathrm{~h} / 24$ et interfacé avec le site Internet de l'entreprise.

- Les candidats accèdent à la liste des offres de l'entreprise sur internet.

- Ils répondent par mini formulaire ou déposent une candidature spontanée.

- Les recruteurs visualisent les réponses aux offres et accèdent au vivier de candidats.

Assurant ainsi la création, modification et publication des offres, puis fournissant les statistiques du nombre de consultations et de réponse pour chaque offre, les solutions permettent la gestion des réponses avec envoi des courriers de réponse aux candidats de façon automatique. Concernant les candidats, les solutions permettent la visualisation, création, et modification des candidats et de leurs pièces jointes et la constitution de base de données « vivier candidats » avec des possibilités de recherche par profils, par compétences et par 
pièces jointes. Les principaux éditeurs spécialisés se sont associés pour créer l'UNESSOR (Union nationale des éditeurs spécialisés en système d'optimisation du recrutement) :

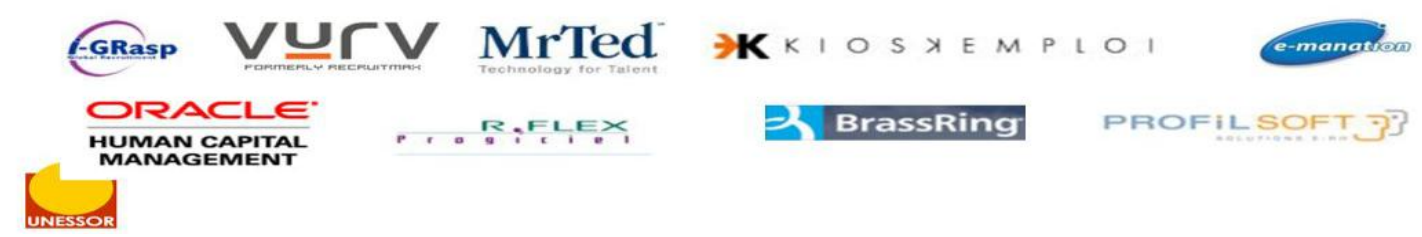

\section{La Rémunération globale :}

Le but de ces solutions informatiques est d'aider les entreprises dans la gestion de la conception, la mise en place et le pilotage d'une politique de rémunération efficace.

Sans une politique de rémunération optimale tenant compte des réalités du marché, il est difficile d'attirer, motiver et garder les meilleurs collaborateurs. Pour ne pas se laisser distancer par la concurrence et face à la course aux talents, mais aussi pour minimiser le turnover, contrôler la masse salariale et éviter le gaspillage de processus de recrutement et de formation de plus en plus coûteux, chaque entreprise se doit réfléchir à la mise en place d'une stratégie RH pertinente et d'une politique de rémunération performante. Face aux éditeurs généralistes qui s'appuient principalement sur les modules paie et reporting, en les croisant avec des enquêtes de rémunération de cabinets (Hewitt, Mercer...) pour permettre la gestion de la politique de rémunération, d'autres éditeurs proposent eux des outils de niches. Ils sont composés de modules qui permettent d'analyser, piloter et contrôler la rémunération des salariés, de réaliser une partie du contrôle de gestion social, de piloter la masse salariale, de préparer les NAO (Négociation annuelle obligatoire) et de mettre en œuvre des opérations de communication sociale (par exemple, de type BSI : bilan social individualisé).

\section{Formation :}

Le module formation a pour finalités de pouvoir créer des éléments (stages, sessions) avec des objectifs individuels et globaux, afin de préparer un budget de formation avec uniquement un volume de participants.

La formation fait parti des processus anciens. Le module existait déjà dans le modèle des SIRH des années 1990.

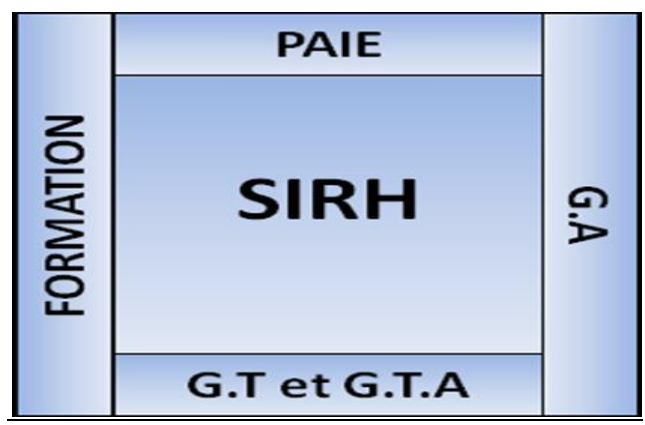

Les fonctionnalités de base permettaient alors d'enregistrer les stages, les sessions et d'inscrire des salariés à des formations. Cependant, ces fonctions sont parfaitement réalisées par un tableur. C'est pourquoi les éditeurs se sont rapprochés de leurs clients et ainsi, ont commencé à proposer la prise en compte de la déclaration 2483, des systèmes d'évaluation des formations, la gestion du prévisionnel et du réalisé, le montage du plan de formation et le suivi des coûts associés.

\section{Tableaux de bord RH :}

La notion de tableau de bord n'est pas propre aux ressources humaines. L'objectif du tableau de bord est de permettre de visualiser, d'un seul coup d'œil, une série de données définies comme étant stratégiques. Il permet de mesurer l'impact des actions entreprises et de dégager les évolutions prévisibles. Un tableau de bord est un outil de pilotage et d'aide à la décision à destination des responsables, mettant en évidence des écarts entre une situation prévue et une situation réelle. De cet écart, naît la mise en place de solutions (actions correctives) par les responsables. Les outils de reporting RH s'adressent principalement, au travers d'états, de rapports, de tableaux de bord, à la DRH qui va les utiliser pour des synthèses, des rapports obligatoires, des analyses prévisionnelles. Mais ils s'adressent aussi aux managers afin de leur fournir, au niveau de leurs services, les tableaux de bord GA-GTA (effectifs, absentéisme, entrées/sorties, heures supplémentaires) et productivité (heures / production). Enfin, ils peuvent également s'adresser aux salariés avec des fiches de synthèse (récapitulatif de carrière, historique des formations suivies ...). Pour le professionnel RH, ils vont donner des 
éléments d'analyse statistique sur la masse salariale, les indicateurs sociaux (absentéisme, licenciement, démission), les temps et activités, les actions de formation, les recrutements.

L'essentiel de l'apport de ces outils d'analyse réside dans le fait d'être les instruments d'une visualisation ordonnée et pertinente d'un certain nombre de priorités et de données de gestion de la stratégie RH, au service de la stratégie globale de l'entreprise. En effet, de tels outils, bien utilisés, contribuent à dynamiser le système dans son ensemble et tendent, de ce fait, à rendre la GRH plus stratégique.

\section{A quoi sert un tableau de bord RH ?}

En ressources humaines comme dans les autres domaines, la qualité des décisions est dépendante de la qualité des informations détenues. Pour construire une politique des ressources humaines efficace et dynamique, il est donc primordial de se doter d'outils performants permettant de faire circuler l'information.

Le tableau de bord ressources humaines est encore fort peu utilisé, en dehors des traditionnelles statistiques d'effectifs, de rotation du personnel et d'absentéisme, cet instrument est portant appelé à un très rapide développement.

\section{Un outil d'aide à la gestion et à la décision:}

C'est un outil d'aide à la gestion, conçu pour analyser la performance. Revenons à la définition du tableau de bord et aidons- nous de l'analogie du tableau de bord automobile. Pour conduire une auto, on a besoin de voir la route et de disposer d'un certain nombre d'indicateurs nous aidant à appréhender les difficultés à venir. L'ensemble de ces instruments nous permet de rester maîtres de notre véhicule.

Seriez-vous prêt à prendre place dans une automobile pour un parcours de montagne avec un chauffeur qui ne quitterait pas des yeux la jauge de carburant et le totaliseur kilométrique ? Sa seule préoccupation étant de respecter ses prévisions de consommation. Non? C'est pourtant ainsi que sont conçus la grande majorité de tableau de bord en usage dans l'entreprise !

Le tableau de bord est ainsi, indispensable à la bonne gestion de l'ensemble des moyens dont dispose le responsable d'une entreprise, d'un département ou d'une unité de travail pour accomplir ses missions.

C'est un outil d'aide à la décision, conçu pour atteindre les objectifs.

Le tableau de bord donne des informations sur les points-clés de la gestion et sur ses dérapages possibles mais il doit surtout être à l'initiative de l'action.

La connaissance des points faible doit être obligatoirement complétée par une analyse des causes de ces phénomènes et par la mise en œuvre d'actions correctives suivies et menées à leur terme.

Ce n'est que sous ces conditions que le tableau de bord peut être considéré comme une aide à la décision et prendre sa véritable place dans l'ensemble des moyens du suivi budgétaire. Il permet aux décideurs enfin de prendre des décisions efficientes, efficaces et justes basées sur des informations pertinentes.

\section{Le tableau de bord $(\mathrm{RH})$ : indicateurs clé des RH :}

$\mathrm{Au}$ niveau RH, la notion de tableau de bord a beaucoup plus de difficultés à s'imposer. Les statistiques généralement élaborées (effectifs, cout du personnel, rotation, absentéisme) sont certes utiles, mais ne constituent pas des indicateurs de pilotage pertinents.

L'importance clé du facteur humain dans l'entreprise réclame la construction d'indicateurs RH pertinents pour le pilotage de la performance de l'entreprise. Ces indicateurs permettront de déterminer les priorités d'action, pour corriger ou anticiper les problèmes, et d'évaluer correctement le succès des programmes mis en œuvre.

\section{Un outil de pilotage :}

Le tableau de bord en tant qu'outil de pilotage, présente plusieurs intérêts. Il permet en effet de :

$\checkmark$ Construire les bases d'un système de management stratégique intégré afin de :

* Clarifier et rendre lisible la stratégie de l'organisation ;

* Communiquer la stratégie à tous les niveaux de l'organisation ;

* Rendre cohérent les objectifs des services et des individus avec la stratégie globale ;

* Relier les objectifs stratégiques à la programmation et à la budgétisation ;

* Procéder périodiquement à des analyses et évaluations de performance.

$\checkmark$ Mettre en cohérence ses processus managériaux;

$\checkmark$ Faciliter la mise en œuvre de la stratégie à long terme par l'ensemble de l'organisation.

On peut retenir là que le tableau de bord RH synthétise les informations propres à un responsable RH, dans ce sens il constitue un outil de pilotage et non du contrôle, parce qu'il permet à un responsable d'évaluer ses performances et de piloter ses propres actions pour améliorer les dits performances en se référant aux différents clignotants clés, des données opérationnelles quantitatives et qualitatives, chiffrés ou non et qui porte sur l'entreprise et son environnement 


\section{- Les axes clés de pilotage pour une politique des RH dynamique}

L'identification de tels indicateurs est beaucoup moins aisée que dans les autres domaines, et ceci pour plusieurs raisons : la complexité des facteurs en jeu ; la difficulté à modéliser précisément les interrelations entre action RH et résultat ; culture de l'entreprise et ses valeurs ;... etc. Les axes d'investigation tournent autour de l'implication des collaborateurs, de l'environnement du travail, du degré d'adhésion à la stratégie de confiance dans les dirigeants, ainsi que l'esprit qualité et client qui prédomine dans l'organisation. La construction de tels indicateurs dont l'évaluation est à suivre dans le temps nécessite la réalisation d'enquêtes périodiques faibles sur les perceptions que les collaborateurs ont des domaines principaux définis et de l'incidence que ces perceptions ont sur leur comportement. En ce qui concerne la réalisation de telles enquêtes, une démarche très professionnelle, et similaire sur plusieurs points à celle d'enquêtes sur le climat social, est à recommander. Les facteurs appréhendés par les indicateurs figurant dans un tableau de bord doivent avoir influence claire sur le succès économique de l'entreprise. Ils servent alors à piloter l'entreprise, notamment à fixer des objectifs et à évaluer les performances. Les indicateurs à construire en la matière doivent refléter le degré d'implication et d'adhésion des collaborateurs à leur organisation et à sa stratégie. Il s'agit donc de réaliser dans ce but des enquêtes bien structurées de perception.

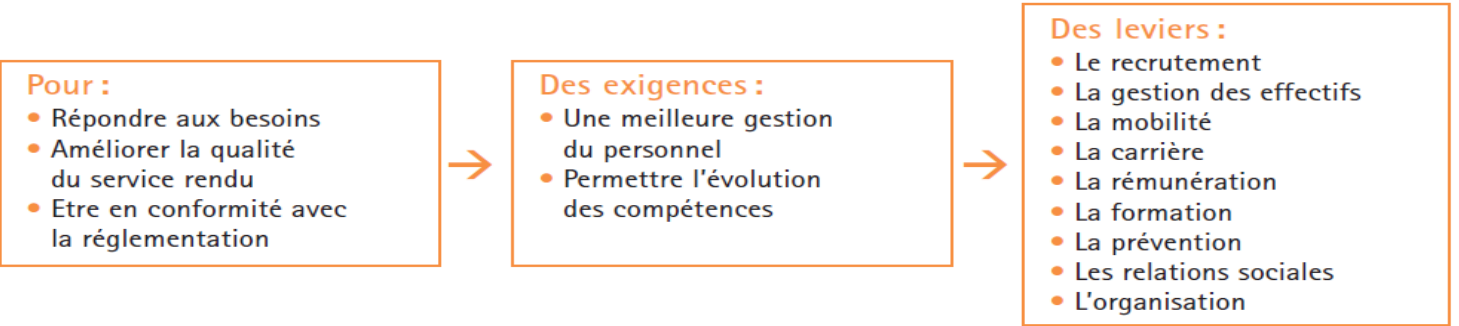

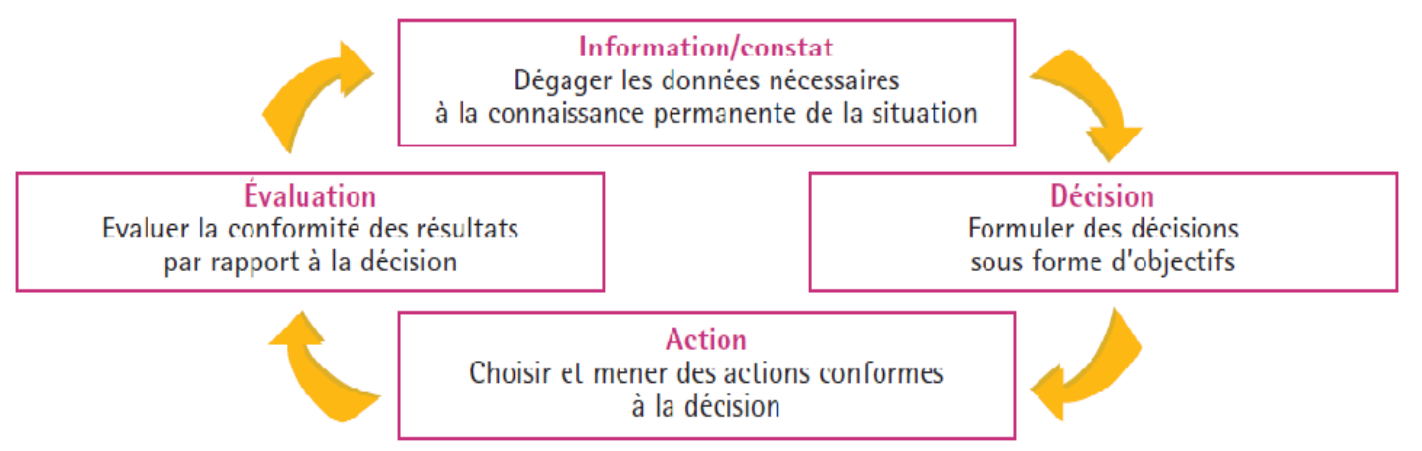

Définition du système de pilotage : un système de pilotage est l'agrégation d'indicateurs qui permet de dégager l'information nécessaire à la prise de déc

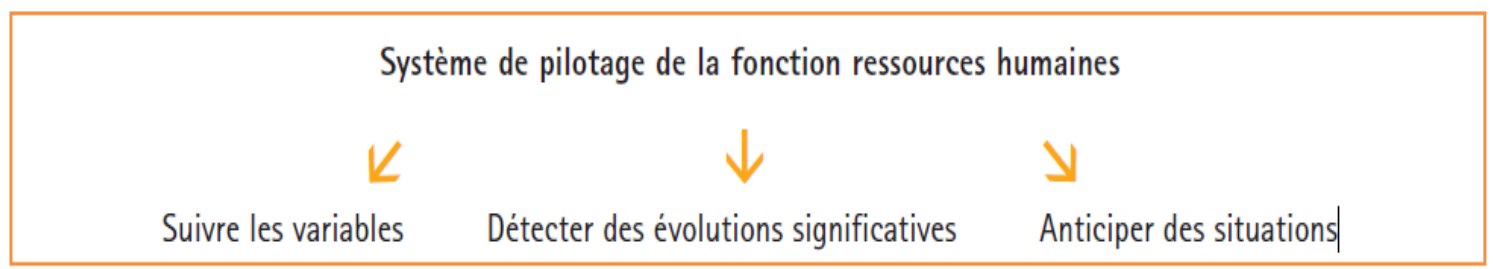

Pour mettre en place un système de pilotage de la fonction ressources humaines, il convient de mettre en place des outils de pilotage. Pour cela il est nécessaire :

- d'identifier les principales missions concernées,

- de préciser les résultats attendus,

- de définir les informations et les indicateurs pertinents nécessaires pour atteindre les objectifs. 


\begin{tabular}{|c|c|}
\hline $\begin{array}{l}\text { Déterminer l'objectif } \\
\text { de chaque indicateur } \\
\text { - délimiter les frontières } \\
\text { de l'observation } \\
\text { (l'objet, les agents concernés, } \\
\text { le service) } \\
\text { - définir la durée } \\
\text { de l'observation } \\
\text { - définir le sens de l'évolution } \\
\text { attendue (augmenter, } \\
\text { diminuer, maintenir) } \\
\text { - quantifier la mesure }\end{array}$ & $\begin{array}{l}\text { Élaborer le tableau } \\
\text { de bord } \\
\text { - élaborer les indicateurs } \\
\text { - anticiper et dégager } \\
\text { les tendances } \\
\text { - croiser les indicateurs } \\
\text { et désigner les corrélations }\end{array}$ \\
\hline
\end{tabular}

\begin{tabular}{|c|c|}
\hline \begin{tabular}{|l|} 
Repérer les sources \\
d'informations \\
- les sources d'informations \\
internes (base de données du \\
personnel, des autres services \\
fonctionnels, des services \\
opérationnels, les études, \\
les enquêtes...) \\
- les sources d'informations \\
externes (centres de gestion, \\
autres collectivités, guide des \\
ratios, études et enquêtes \\
des organismes de conseils).
\end{tabular} & $\begin{array}{l}\text { Présenter les outils } \\
\text { de pilotage pour une } \\
\text { communication efficace } \\
\text { Le choix de la forme de } \\
\text { présentation dépend: } \\
\text { - de la cible } \\
\text { - du message à faire passer } \\
\text { - de l'effet escompté } \\
\text { - des règles de communication } \\
\text { courante }\end{array}$ \\
\hline
\end{tabular}

Lorsque les objectifs et le champ de l'observation ont été déterminés, il est important de repérer les données disponibles à la DRH, mais aussi dans les services et directions. Les résultats de l'observation doivent être disponibles à l'échelon pertinent : certains indicateurs sont suivis au niveau de la DG et de la DRH, d'autres sont utiles aux différents niveaux des services opérationnels. Ils aident les encadrant de proximité et les responsables de services à jouer tout leur rôle Lorsque les objectifs et le champ de l'observation ont été déterminés, il est important de repérer les données disponibles à la DRH, mais aussi dans les services et directions. Les résultats de l'observation doivent être disponibles à l'échelon pertinent : certains indicateurs sont suivis au niveau de la DG et de la DRH, d'autres sont utiles aux différents niveaux des services opérationnels. Ils aident les encadrant de proximité et les responsables de services à jouer tout leur rôle.

\section{CONCLUSION}

L'objectif de cet article était d'apporter un éclairage sur le système d'information des ressources humaines (SIRH) qui est devenu un outil incontournable des professionnels de la gestion des ressources humaines (GRH), et qui est la base pour l'élaboration d'un tableau de bord RH parce qu'il il constitue pour les responsables $\mathrm{RH}$ et les décideurs un outil de pilotage et d'aide à la prise de décisions. Le management des ressources humaines est au centre du dispositif économique, responsable de l'actif qui régénère tous les autres. Il est temps pour la fonction RH de sortir de son rôle technique et administratif pour devenir un partenaire stratégique, contribuant, au même titre que les autres, aux résultats économiques et sociaux de l'entreprise et oriente la pensée des décideurs pour la prise de bonnes décisions à l'aide d'un SIRH et des tableaux de bord RH. La démarche décrite dans cet article met un accent particulier sur la mise en place d'un système de pilotage des ressources humaines révélateur de la performance des processus RH et de l'adaptation du capital humain s'impose comme une nécessité. En s'impliquant dans le processus de pilotage de l'entreprise, la DRH démontre sa contribution à la création de valeur économique et favorise la prise en compte des indicateurs de création de valeur intellectuelle et sociale.

Les plans d'action RH alignés sur les orientations stratégiques de l'entreprise, cohérents entre les différentes fonctions RH et les entités opérationnelles, s'intègrent dans la chaine de création de valeur globale. L'évaluation des résultats RH à court terme s'exerce dans le souci de préserver et préparer la performance du capital humain à moyen et long terme. Le développement du capital humain se réalise au jour le jour et dans la durée, le système de mesure traduit cette double nécessité. Pour apporter une réelle contribution à la création de valeur, les RRH, tout en maintenant un niveau d'expertise technique indispensable à leur professionnalisme, doivent s'ouvrir à la culture économique et financière de l'entreprise et développer une approche entrepreneuriale. La démarche de pilotage requiert des qualités de leader pour convaincre du bien-fondé des actions RH, de gestionnaire pour optimiser les investissements, d'entrepreneur pour innover et de stratège pour intégrer les évolutions de l'environnement. Le poste de DRH peut ainsi préparer à des missions de direction opérationnelle ou générale. Le passage par une fonction RH centrée sur la création de valeur globale constitue une étape essentielle à la préparation des futurs cadres dirigeants. À l'inverse, il serait souhaitable que les parcours de carrière des RRH passent par des missions opérationnelles et l'exercice du management de proximité.

Le positionnement stratégique et économique de la fonction $\mathrm{RH}$ implique de nouveaux modes de fonctionnement et de nouvelles compétences des professionnels RH :

* Mieux formés à la gestion économique et financière, il leur sera plus facile de modéliser et chiffrer le résultat des actions engagées pour développer le capital humain. Une organisation moins cloisonnée des services RH est indispensable à la mobilisation des équipes RH autour des objectifs communs de création de valeur. 
+En démontrant leur performance, la démarche de pilotage facilitera le déploiement et l'appropriation des outils RH par le management de proximité. Aucun progrès ne pourra se faire sans qu'ils soient convaincus de l'intérêt des processus et mieux préparés à manager leurs équipes.

\#Un travail étroit avec la direction financière permettra, principalement dans la phase de lancement, de valider la pertinence des indicateurs RH et des modes de calcul.

\# Enfin, le SIRH devra s'organiser et développer les moyens adaptés au pilotage de la fonction.

\section{Webographies :}

\section{$\underline{\text { Références }}$}

- http://fr.slideshare.net/aaitomar/cours-sirh-chapitre-ii

- http://www.officielrh.com/ressources_humaines/detail_dossier_ressources_humaines php?rub $=146 \&$ ssrub $=148 \&$ dossid $=8$

- http://www.marocentreprise.com/rh/1377-un-systeme-dinformation-ressources humaines-sirh.html

- http://danielus.over-blog.com/article-pourquoi-un-systeme-d-information-des 47420870.html

- http://www.lavieeco.com/news/la-vie-eco-carrieres/concevoir-un-tableau-de-bord-rh-12509.html

- http://www.atlasdumanagement.com/index.php?article=99\&zone=2

- http://books.google.co.ma/books?id=WBGiCC W7yQC\&pg=PA175\&lpg=PA175\&dq=le+tableau+de+bord+ prospectif+ressources+humaines\&source=bl\&ots=LqsK3gCTy2\&sig=TJnUCQdfw76 ESpC33xhojB7Sbw\&

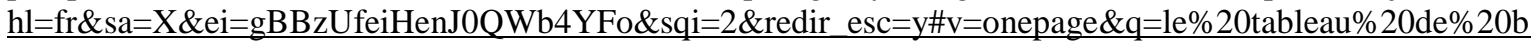
ord\%20prospectif\%20ressources\%20humaines\&f=false

- http://www.ogexo.fr/tableaux-de-bord-rh-principes-delaboration-et-dimplementation/

\section{Bibliographies :}

- Le tableau de bord RH «construire, mettre en œuvre et évaluer le système de pilotage », Joëlle Imbert, Editions d'organisation ;

- Management - ressources humaines, le SIRH, Michelle Gillet Patrick Gillet, édition DUNOD ;

- Le SIRH : un atout dans l'optimisation de la GRH au service de l'entreprise, Gilles Exbrayat Nathalie Fisteberg Octobre 2010 ;

- Tableaux de bord outils de performance, Denis Molho Dominique Fernandez-Poisson, EYROLLES Editions d'organisation ;

- Tableaux de bord de gestion et indicateurs de performance, Pierre Voyer, $2^{\text {ème }}$ édition,

- Mémoire de fin d'étude, tableau de bord un outil de performance et d'aide à la décision, Ghani Khadija,2009 ;

- Pas de DRH sans SIRH, Bernard Just, Editions Liaisons 2010; 